\section{$\underset{\text { hommes }}{\text { \& migrations }}$}

\section{Hommes \& migrations}

Revue française de référence sur les dynamiques

migratoires

1313 | 2016

1983, le tournant médiatique

\title{
La victoire de Yannick Noah à Roland-Garros
}

Ou le rêve déçu d'une icône antiraciste

\section{Stéphane Mourlane et Philippe Tétart}

\section{OpenEdition \\ 1 Journals}

\section{Édition électronique}

URL : http://journals.openedition.org/hommesmigrations/3571

DOI : 10.4000/hommesmigrations.3571

ISSN : 2262-3353

\section{Éditeur}

Musée national de l'histoire de l'immigration

\section{Édition imprimée}

Date de publication : 1 janvier 2016

Pagination : 105-112

ISBN : 978-2-919040-34-6

ISSN : 1142-852X

Référence électronique

Stéphane Mourlane et Philippe Tétart, "La victoire de Yannick Noah à Roland-Garros », Hommes \& migrations [En ligne], 1313 | 2016, mis en ligne le 01 janvier 2019, consulté le 16 mars 2020. URL: http://journals.openedition.org/hommesmigrations/3571; DOI : https://doi.org/10.4000/ hommesmigrations.3571 


\title{
LA VICTOIRE DE YANNICK NOAH À ROLAND-GARROS OU LE RÊVE DÉÇU D'UNE ICÔNE ANTIRACISTE
}

\begin{abstract}
par STÉPHANE MOURLANE, maître de conférences en histoire contemporaine à l'université d'Aix-Marseille, UMR 7303 Telemme, et PHILIPPE TÉTART, maître de conférences en histoire contemporaine à l'université du Maine, VIPS - EA4636.
\end{abstract}

\author{
Le 5 juin 1983, la victoire de Yannick Noah à Roland-Garros fait \\ la joie de la France entière. En pleine crise économique \\ et sociale, confronté à la fois à la récession et à la montée \\ du racisme que dénoncent les enfants d'immigrés, le pays s'est \\ trouvé une icône. Si l'embrasement médiatique fait de Noah \\ le nouveau visage d'une société réconciliée avec elle-même, \\ le joueur lui-même paraît réticent à endosser le symbole. \\ Une manière d'échapper aux assignations identitaires qui \\ surdéterminent son exploit sportif.
}

La victoire de Yannick Noah aux Internationaux de France de tennis est assurément l'un des grands événements de l’année 1983. Un événement sportif tout d'abord. Le 5 juin, le joueur met fin "aux années de vaches maigres du tennis français ${ }^{1 \text { ". }}$ Aucun Français n'a remporté le tournoi depuis la victoire de Marcel Bernard en 1946. Plus largement, aucun ne s'est jamais imposé dans l'un des trois autres tournois dits du "Grand Chelem" ». Enfin, sur une échelle de temps plus longue, personne n'a jamais brillé comme les Mousquetaires, vainqueurs à six reprises de la Coupe Davis entre 1927 et 1932. Aujourd'hui encore, la place occupée par cette victoire dans la mémoire collective
- il est vrai entretenue par la manie commémorative des médias, par la carrière de chanteur de l'exjoueur, ses actions caritatives et son retour régulier sur les terrains ${ }^{3}$ - souligne avec force l'importance de ce haut fait, tant en 1983 que dans le cadre plus large de l'histoire sportive tricolore. Un événement médiatique ensuite, vécu sur le mode de l'émotion partagée. Temps fort du calendrier sportif, RolandGarros, diffusé en direct depuis 1978, est devenu en peu de temps une grande messe sportive cathodique dont la capacité de séduction est notamment liée à son statut de laboratoire de la télévision sportive ${ }^{4}$. L'émotion suscitée par la victoire est donc d'autant plus forte que les téléspectateurs peuvent 
avoir le sentiment d'être les témoins intimes et privilégiés d'un mythe en train de se forger ${ }^{5}$. Ce 5 juin précisément, 11 millions d'entre eux sont rivés devant leur téléviseur pour suivre la finale entre le Suédois Mats Wilander et Yannick Noah. Audience record pour un après-midi dominical. L'émotion est à son comble lorsque, aussitôt après la balle de match, Noah père et fils, faisant fi du protocole tennistique policé, se ruent l'un vers l'autre et s'étreignent au milieu du

Dans un tel climat, l'élévation

au rang de héros national

d'un sportif aux origines

mêlées - selon une formule

volontiers reprise par

les médias - revêt une forte

portée symbolique

qui n'échappe ni au public,

ni aux commentateurs,

ni au champion lui-même. court central. Le lendemain, la presse sportive se régale de cette étreinte vouée à la postérité et de l'émotion livrée sans fard par Yannick Noah. « 17 h 34, la France est coupée en deux. La moitié pleure devant sa télé. L'autre moitié a les larmes aux yeux ${ }^{6} "$, liton dans L'Équipe. Le reste de la presse est au diapason : «Fantastique Noah » (Le Figaro), « Les Sept Glorieuses » (France Soir), « Le Bonheur de RolandGarros » (L'Humanité). Même Le Monde et Libération, pourtant peu diserts et souvent critiques en matière sportive, succombent. Le premier y va par une référence au héros de Conan Doyle : «Évidemment mon cher Noah". Le second lance "Noah is beautiful » - adaptation d'une tocade Belle Époque muée en mot d'ordre militant: «Black is beautiful».

\section{Les sens d'une victoire}

Cet événement marquant sur le plan sportif, et médiatique donc, l'est aussi sur le plan social et politique, car la victoire de Yannick Noah souligne aussitôt la problématique de l'altérité. Elle le fait de façon d'autant plus notable que, d'une part, le joueur a été à plusieurs reprises l'objet de manifestations de rejet dont la presse s'est émue (notamment d'insultes racistes lors d'un tournoi argentin en 1982) et que, d'autre part, il lui arrive de parler de sa négritude sur un mode identitaire et revendicatif (" Je serai l'ambassadeur des Noirs. Je leur prouverai qu'un Noir peut battre un blanc $\left.{ }^{7} »\right)$. Les tensions politiques et sociales liées aux questions de l'immigration et du racisme, rémanentes depuis un siècle, sont alors très vives ${ }^{8}$. Au printemps 1983, une fois de plus, le débat s'envenime. En mars, la percée électorale du Front national lors du scrutin municipal de Dreux est l'un des principaux facteurs qui relancent le débat sur le thème de l'identité française menacée 9 . À l'Assemblée et par médias interposés, droite et gauche ferraillent souvent sur cette question.

Dans un tel climat, l'élévation au rang de héros national d'un sportif aux origines mêlées - selon une formule volontiers reprise par les médias revêt une forte portée symbolique qui n'échappe ni au public, ni aux commentateurs, ni au champion lui-même. "Qui peut oublier, au lendemain de la finale des Internationaux de France de tennis, que Yannick Noah, fils de Zacharie, est aussi un Noir ? » écrit Jérôme Bureau dans L'Équipe ${ }^{10}$.

Au printemps 1984, dans un reportage diffusé sur Antenne 2 par le magazine Résistances, Tahar Ben Jelloun revient sur cette question en commentant des images de la victoire de Yannick Noah, du rugbyman Serge Blanco et du footballeur Marius Trésor inscrivant un but lors de la demi-finale de la Coupe du monde de football 1982 contre l'Allemagne. Il dit : "La France minoritaire du racisme, tout en réclamant le renvoi des immigrés chez eux, oublie des fois d'être raciste. Elle fait des exceptions sur des visages, des noms, des vainqueurs. Ils n'ont pas la peau qu'il faut et pourtant on oublie ces détails dès qu'ils gagnent, dès qu'ils font gagner la France ${ }^{11}$. » En

5. Georges Vigarello, Du jeu ancien au show sportif. La naissance d'un mythe, Paris, Seuil, 2002, p. 161. 6. Ibid. 7. L'Équipe, 27 novembre 1982. 8. Voir notamment Gérard Noiriel, Immigration, antisémitisme et racisme en France (XIXe-XXe siècle). Discours publics, humiliations privées, Paris, Fayard, 2007 ; Laurent Dornel, La France hostile. Socio-histoire de la xénophobie 1870-1914, Paris, Hachette, 2004 ; Ralph Schor, L'Opinion française et les Étrangers en France, 1919-1939, Paris, Publications de la Sorbonne, 1985 ; Yvan Gastaut, L'Immigration et l'Opinion en France sous la V' République, Paris, Seuil, 2000. 9. Voir Gérard Noiriel, Population, immigration et identité nationale en France (XIXXXXe siècle), Paris, Hachette, 1992. 10. L'Équipe, 6 juin 1983 11. INA, CAB8400015001: Ben Jelloun Tahar, "Racisme décaféiné ", in Résistances, Antenne 2, $1^{\text {er }}$ mars 1984. 
quelques mots, l'écrivain rappelle la schizophrénie d'une partie de l'opinion, celle-là même qui porte Yannick Noah à se sentir français dans la victoire et camerounais dans la défaite et, quelques années après sa victoire, à avoir ce mot : "Quand on perd, la couleur redevient un défaut ${ }^{12}$. "

Les enjeux liés à cette victoire dépassent donc la seule personne de Yannick Noah. Son personnage, ses origines, catalysent en effet un débat qui renvoie à la question de l'immigration et du racisme. Les pages qui suivent se proposent d'observer la façon dont, dans le contexte spécifique de 1983, la presse et la télévision jouent avec la figure symbolique de Yannick Noah.

\section{Un héros et une vedette}

Dès le début de sa carrière, Yannick Noah fait l'objet d'une attention médiatique soutenue ${ }^{13}$. En 1978, son émergence au plus haut niveau suscite aussitôt un fantasme : qu'il réveille la belle endormie - la légende tennistique française - en remportant un Grand Chelem. Cette aspiration est d'autant plus forte que le numéro un français du moment, François Jauffret, à 36 ans, est un vétéran, et que les autres joueurs en vue - Patrick Proisy, Christophe Roger-Vasselin, Gilles Moretton, Pascal Portes, Dominique Bedel - ne brillent pas au plan international. Bref, Yannick Noah peut combler un vide et redorer la saga sportive tricolore. Très vite associé à la mémoire des Mousquetaires et de Suzanne Lenglen, on le voit comme un héritier, celui qui saura mettre un terme à la longue attente d'une victoire masculine à Paris, celui qui saura redonner sa fierté à la nation.

Cet espoir a pour socle les qualités qu'on lui prête alors : sérieux, modestie, lucidité, enthousiasme, ambition, générosité dans l'effort et, surtout, franchise et maturité. Ces qualités, lit-on souvent, doivent être mises au service de la France. Dès la fin des années 1970, il est donc investi d'une mission de représentation. Yannick Noah, lit-on dans La Vie, dès 1978, "représente la France ${ }^{14}$ ». Et le joueur lui-même semble assumer cette responsabilité. En 1979, lors d'une rencontre de Coupe Davis contre la Suisse, affichant une posture ouvertement patriote, il s'étonne que l'un de ses adversaires, le Suisse Heinz Günthardt, «représente son pays (...) avec autant de passivitét $e^{15}$. Trois ans plus tard, il déclare à L'Équipe qu'il est conscient de sa « responsabilitét ${ }^{16}$ ». La plupart des journalistes font de Yannick Noah ce héros généreux dans la bataille, un héros d'autant plus sympathique que, tout combattant qu'il soit, il semble pétri d'humanité. Il apparaît comme l'antithèse d'Yvan Lendl ou de Björn Borg, présentés comme de froides machines à relancer la balle. Lui ne sera jamais " un robot (...) même s'il devient numéro un ${ }^{17}$ ». Au final, il semble proche du commun des mortels; et cela est une autre part de l'étoffe héroïque : être comme les autres et unique à la fois, ce par quoi l'idée du modèle est plus forte encore.

L’héroïsme de Yannick Noah se nourrit d'une dernière facette : son vedettariat. Les enjeux liés à cette victoire dépassent donc la seule personne de Yannick Noah. Son personnage, ses origines, catalysent en effet un débat qui renvoie à la question de l'immigration et du racisme. Sous ce jour, il est sans doute, en France, la figure pionnière de la peopolisation des sportifs, une procédure tenant aujourd'hui de l'ordinaire, voire de la norme. En 1983, il est donc un héros et une vedette qui participe aux premiers prime time télévisés. Les performances sportives ayant façonné sa première notoriété se doublent dès lors, selon le sociologue Patrick Mignon, d'une "mise en scène de soi qui permet la gestion d'un capital de célébrité ${ }^{18}$ ". La presse s'intéresse aussi bien à son tennis quaux 
autres aspects de sa vie : son projet de mariage, ses incursions dans la publicité et dans le monde de la variété, où il se montre sous le jour d'un jeune homme aimant chanter, danser, jouer la comédie. L'aisance qu'il y montre déconcerte. On a tôt fait de l'assimiler à une star ${ }^{19}$.

Partant de ce terreau, on comprend bien que, dans le tourbillon médiatique consécutif à la victoire du 5 juin 1983, celle-ci soit vécue sur le mode de la " consécration ${ }^{20}$ ". Les

Ce fantasme médiatique du déracinement prend probablement sa source dans le fait que Yannick Noah

exprime souvent son attachement, matriciel, pour le Cameroun. médias, unanimes, saluent le « héros ${ }^{21}$ » ou, mieux encore - selon l'expression de la journaliste Christine Ockrent dans le journal télévisé au soir de la victoire -, le « héros national ». À l'heure du déjeuner, lors du journal télévisé d’Antenne 2, le présentateur Daniel Bilalian reprend la formule de L'Équipe et ajoute que la victoire de Yannick Noah " dépasse le cadre strictement sportif22». Quelques semaines après le tournant de la rigueur décidé par le gouvernement socialiste (23 mars) pour faire face à la détérioration de la situation économique, le ministre de l'Économie et des Finances Jacques Delors déclare à son tour : "Il faut que la France se batte comme Noah. » L'Équipe en fait "l'homme le plus aimé et le plus convoité de France », un "rassembleur ${ }^{23}$ ». Le quotidien s'aventure même à publier cette assertion : "Pour cause de gloire médiatique, Noah, offert à la reconnaissance nationale et au patrimoine, n'est plus un "grand noir"24".

\section{Du rappel des origines aux représentations exotisées}

Rien n'est moins sûr pourtant car l'entrée du joueur sur les radars médiatiques va d'emblée de pair avec la question de ses " origines mêlées ».

Dès 1979, on l'interroge sur la façon dont il appréhende son métissage. En la matière, Noah se plaît à semer l'ambiguïté. Il prend volontiers un accent africain caricatural, le même que celui de Michel Leeb, humoriste en vogue. Lorsqu'on l'interroge sur ses origines, sur la négritude, ses réponses se partagent entre facéties, autodérision et provocation. En 1981, il publie une première autobiographie dans laquelle on lit: "Si j'ai choisi la nationalité française, c'est pour mon métier... Cette deuxième nationalité est, en fait, le résultat d'une situation professionnelle ${ }^{25}$ ». On ne saurait tout à fait accréditer cet utilitarisme tant ses positions sont versatiles. Reste que ce type de déclaration propage l'idée d'une identité flottante, partagée, voire fracturée. Cette dimension appelle un court aparté biographique. Yannick Noah est né en France, en 1960, à Sedan. Son père, Zacharie, Camerounais, est arrivé en France en tant qu'étudiant avant de devenir footballeur professionnel au sein du club local. Sa mère, Marie-Claire, est française. Sur le plan juridique, la question de sa nationalité ne se pose pas. En vertu du droit du sol et du droit du sang inscrits dans la loi depuis $1889^{26}$, il est français. Et pourtant, la référence au pays paternel, où il a vécu entre 3 et 11 ans, est constante, à la fois sous la forme d'une revendication (le joueur s'y réfère et parle avec émotion, par exemple, de son grandpère) et d'une assignation (la presse se complaisant dans la " camerounisation » du joueur). À la charnière des deux se dessine une image, souvent reprise, comme dans Le Monde : celle du "déraciné27 ", de celui qui a " quelque peine à faire l'aller et retour entre le milieu très clinquant de [sa] vie professionnelle et [ses] origines mêlées ${ }^{28}$ ».

Ce fantasme médiatique du déracinement prend probablement sa source dans le fait que Yannick Noah exprime souvent son attachement, matriciel, pour le Cameroun. À l'occasion d'un séjour qu'il y fait début 1983, il évoque un «retour aux sources ${ }^{29}$ ». Sa coiffure, faite de dreadlocks, ou son habituel 
serre-poignet rouge, jaune et vert, " couleurs de sa patrie paternelle ${ }^{30}$ ", agissent comme des emblèmes de la culture rastafari, du reggae (lequel connaît sa première mode en France depuis la fin des années $1970^{31}$ ) et africaine. Le 5 juin, lors du traditionnel discours sur le court central, après la remise du trophée, Yannick Noah rend d'ailleurs un vibrant hommage au Cameroun. Mais de déracinement il n'est point question dans les propos du joueur, et la presse, souvent, bascule dans la surinterprétation. La victoire de Roland Garros fait donc plus que jamais ressortir la négritude du joueur. À la rubrique «signes particuliers », Le Quotidien révèle cette tendance : s'il adore la musique et les copains, il est d'abord «noir ${ }^{32}$ ». Lui-même, mi-médusé miexaspéré, finit par le dire : «Il reste que je suis noir ${ }^{33}$. " À l'évidence, le récit médiatique laisse la place aux préjugés racialistes d'inspiration colonialiste et post-colonialiste qui, de longue date, décrivent les qualités physiques des sportifs noirs ${ }^{34}$. L'animalité du corps en est l'un des traits soulignés ${ }^{35}$. Et Yannick Noah n'y échappe pas. Noah danse "sensuel, félin et charmeur dans les nouvelles arènes au bois de Boulogne $e^{36}$ ", lit-on dans le Nouvel Observateur. Dans Le Figaro, Jacques Chancel évoque « un superbe félin », un «fauve $e^{37}$ ». Par allusion au surnom de l'équipe de football du Cameroun, L'Humanité parle du «Lion indomptable de Roland-Garros ${ }^{38}$ ».

Pourtant, et paradoxalement, les journalistes semblent appréhender la double identité du champion comme un symbole et un atout dans le développement du dialogue entre les cultures. Le président François Mitterrand y voit quant à lui l'occasion d'un profit diplomatique dans le cadre de sa politique africaine. Quelques semaines après la finale, il convie le joueur à l'accompagner lors de son voyage officiel à Yaoundé. Les médias sportifs et généralistes y font largement et positivement écho jusqu'à l'automne, tirant tout le suc d'un retour sur les terres d'origine présenté sur le mode du pèlerinage.

Enfin, la victoire de Yannick Noah à Roland-Garros est aussi présentée comme celle de l'Afrique. Il hérite là d'une autre responsabilité nationale, au nom de sa "seconde patrie ", le Cameroun et, au-delà, continentale et identitaire, au nom des «noirs ${ }^{39}$ ». Nombre de journaux proposent à leurs lecteurs de suivre l'événement au Cameroun aux côtés de la famille Noah et des Camerounais, "collés à leur transistor ${ }^{40}$ ", pour qui il est, selon le président Paul Biya « un sujet de fiertée $e^{11} »$. Pour Le Nouvel Observateur, " aucune ambiguité n'est plus permise au moment même où le triomphe de Pourtant, et paradoxalement, les journalistes semblent appréhender la double identité du champion comme un symbole et un atout dans le développement du dialogue entre les cultures.

Noah délivrait les foules d'une tension éperdue. Car il fallut bien voir alors le jeune héros se fondre dans les étincelantes noirceurs de l'effusion paternelle. L'Afrique reprenait possession des siens ${ }^{42}$ ». Quant à Libération, on y lit que sa victoire n'est "pas vraiment une victoire française ", mais celle "d'un champion d'origine camerounaise $^{43}$ ».

La question, complexe, de la négritude, des origines et in fine des responsabilités du joueur traverse donc l'ensemble du discours médiatique. Elle relève du topique. Elle relève aussi de la redite. Depuis les années 1950 en effet, les sportifs d'origine étrangère (de première génération ou non), les footballeurs en particulier, sont souvent vus au prisme d'une revanche à prendre, entre logique de contentieux et principe victimaire, qui, en toute chose, les ramène vers leur couleur, leur culture, la terre natale et la mémoire trouble du colonialisme $e^{44}$. Comme Zinedine Zidane un quart de siècle après lui, on attend de Yannick Noah qu'il 
incarne positivement cette revanche en réussissant le tour de force d'être à la fois un modèle sportif et un héros français, le fier représentant de l’immigration, l'homme de la belle quête des origines et, pourquoi pas, un coambassadeur de la France et de l'Afrique ${ }^{45}$. On l'attend avec une force d'autant plus grande que Yannick Noah chambarde la représentation du tennis. Le seul Noir à avoir brillé avant lui se nomme Arthur Ashe, un Américain. Il a d'ailleurs été son premier mentor après qu'il l'a eu découvert lors d'une exhibition au Cameroun. En somme, Yannick Noah dérange et aimante les interrogations car il vient aussi rebattre les cartes d'un univers tennistique - et de sa représentation médiatique - où joue depuis

Héros français et champion

d'Afrique à la fois, Yannick

Noah semble donc taillé

pour être une figure

biculturelle positive

et de concorde, et, pourquoi

pas, un modèle digne

de la double mythologie de

l'intégration républicaine

et du Paris noir, celui

de Joséphine Baker, de Blaise

Diagne, d’Aimé Césaire...

turelle positive et de concorde, et, pourquoi pas, un modèle digne de la double mythologie de l'intégration républicaine et du Paris noir, celui de Joséphine Baker, de Blaise Diagne, d'Aimé Césaire ${ }^{47} \ldots$ Contexte aidant, il peut aussi apparaître comme une figure antiraciste et sa victoire comme un emblème, un catalyseur. Le contexte invite à une telle projection. Au fil des années 1970, le militantisme antiraciste, porté de longue date par des instances comme la Ligue des droits de l'homme ou le Mouvement contre le racisme et pour l'amitié entre les peuples (MRAP), est monté en puissance. On lui doit pour partie, à la charnière des années 1970-1980, la levée d'une nouvelle génération antiraciste ${ }^{48}$. Mais, en un temps où les discours xénophobes portés par l'extrême droite sont en pleine recrudescence ${ }^{49}$ - les stades, du moins ceux de football, n'y échappent pas ${ }^{50}$-, cette génération réinvente les codes de la prise de parole. Au militantisme un peu suranné des intellectuels de gauche, champions de l'écrit et de l'indignation pétitionnaire, elle préfère un militantisme de l'action, dans l'espace public. Affaire de rupture générationnelle - celle de la télévision contre celle de l'écrit - et de milieu aussi : ceux qui marchent pour l'égalité des chances et contre le racisme, entre octobre et décembre 1983, incarnent un nouvel univers antiraciste en ce qu'ils font l'expérience effective du racisme. Les médias ne s'y trompent pas. Ils suivent la " Marche des Beurs » avec attention ${ }^{51}$

Yannick Noah semble pouvoir être un ambassadeur de l'antiracisme. Et cette assignation se cristallise au lendemain de sa très nette victoire en demi-finale de Roland-Garros contre son compatriote, Christophe Roger-Vasselin. Quelques jours plus tard, Le Nouvel Observateur souligne à quel point la valeur symbolique de cette victoire échappe au joueur, mais la rédaction lui assigne malgré tout une lourde responsabilité : " Ce dont Yannick n'a probablement pas conscience, c'est que par sa présence dans tous les foyers français, une présence associée à une victoire française, dans le rayonnement de sa grâce et la dimension contagieuse de son charme, il aura un instant, et bien plus que d'autres, figé sur place la vague de racisme qui déferle sur le pays ${ }^{52}$. "Si

40. Libération, 7 juin 1983 41. Le Monde, 7 juin 1983.42. Nouvel Observateur, 10 juin 1983. 43. Libération, 7 juin 1983. 44. Stéphane Beaud, Gérard Noiriel, "De l'immigration dans le football », in Vingtième Siècle. $n^{\circ} 26,1990$, p. 96. 45. Pour le cas des footballeurs, Steven Apostolov, Yvan Gastaut, «Zinedine Zidane's return to the land of his ancestors : politics, diplomacy or something else? ", in Soccer \& Society, vol. 15, n 5, 2014, pp. 685-695. 46. Pap Ndiaye, La Condition noire. Essai sur une minorité française, Paris, Calmann-Lévy, 2008, p. 320, et plus généralement pp. 224-237. 47. Voir Pascal Blanchard, Éric Deroo, Gilles Manceron, Le Paris noir, Paris, Hazan, 2001 et Benoît Hopquin, Ces noirs qui ont fait la France, Paris, Calmann-Lévy, 2009. 48. Yvan Gastaut, "Générations antiracistes en France 1960-1990 ", in Cahiers de la Méditerranée, vol . 61, n 1, 2000, pp. 289 -303. 49. Ralph Schor, "Parler des étrangers : les mots du Front national ", in Cahiers de la Méditerranée, vol. 54, n 1, 1997, pp. 117-137. 50. Nicolas Hourcade, "Les expressions racistes des supporters de football français depuis les années 1980 ", in Claude Boli, Patrick Clastres, Marianne Lassus (dir.), Le Sport en France à l'épreuve du racisme, Paris, Nouveau Monde, 2015, pp. 105-116. 
l'on excepte les commentaires proprement sportifs et ceux, nombreux, relatifs à sa vie privée, rares sont alors les écarts à la règle tacite faisant de Yannick Noah la figure emblématique de l'heureux brassage français.

De son côté, le joueur n'apparaît toujours pas enclin à endosser ce rôle de porte-étendard de l'antiracisme. Au printemps 1983, alors que le débat fait rage autour de l'interdiction faite par le gouvernement à la Fédération française de rugby de participer à des rencontres contre l'Afrique du Sud au nom de la lutte contre l'apartheid ${ }^{53}$, Yannick Noah, qui a pourtant par le passé dénoncé la ségrégation raciale sud-africaine brouille les cartes en déclarant qu'il a envie d'aller y jouer ${ }^{54}$. En mai 1983, dans Le Figaro, on lit : «Il refuse, malgré les invitations très fréquentes, de se prononcer sur un sujet - l'existence d'un quelconque racisme à son encontre en France ${ }^{55}$. " Bien plus tard, il dira avoir été victime de racisme dès $1979^{56}$. Longtemps après avoir déclaré qu'il se sentait concerné par les problèmes des Noirs dont il est le «leader un peu malgré lui depuis qu'Ashe s'est retiré des courts ${ }^{57}$ », il devisera aussi sur son statut d' " athlète noir ${ }^{58}$ ». Au début de l'hiver 1983, il avoue avoir hésité à accepter de donner un entretien pour un numéro thématique de L'Équipe Magazine intitulé «Sport et racisme ${ }^{59}$ ». Il considère n'être "représentatif de personne » et ne se voit pas comme un "porte-parole ». Peut-être faut-il chercher la source du détachement de Yannick Noah dans la phase difficile qu'il traverse. Après avoir déjoué une partie de l'été, exténué, il décide de s'expatrier aux ÉtatsUnis. Son départ est vécu comme une trahison, un affront à la nation. "Noah prend la fuite " liton dans L'Équipe ${ }^{60}$. " 54 millions de Noah trahis par Noah » renchérit Le Monde ${ }^{61}$. Six mois après avoir célébré la victoire sans aucun filtre ni recul sur sa signification, une partie des journalistes baignent toujours dans un régime d'extrapolation où la psychologie de comptoir le dispute à la déception, le deuil de l'émotion. Le Monde n'hésite pas à lancer ce jugement : Yannick Noah est en permanence fragilisé parce qu'il n’a pas réussi « la soudure affective entre une culture européenne et des racines africaines ${ }^{62}$ ». Quant au joueur, en réponse aux questions renaissantes sur son identité, balancée entre Cameroun et France, il botte en touche : il «se sent de nulle part $^{63}$ ». Sa double culture, perçue comme une force et comme la source du miracle printanier, redevient un facteur de déséquilibre. Tout
Comme Zinedine Zidane un quart de siècle après lui, on attend de Yannick Noah qu'il incarne positivement cette revanche en réussissant le tour de force d'être à la fois un modèle sportif et un héros français, le fier représentant de l'immigration. autant que "Noah l'Africain » était célébré, Noah le néo-Américain est honni. Dans de telles conditions, le jeune homme - il n'a après tout que 23 ans... - peine évidemment à trouver sa place et le ton juste face à l'épineuse question du racisme.

\section{Conclusion}

La victoire de Noah est un événement majeur dont la temporalité n'est pas circonscrite à l'année 1983. Aussitôt mythifiée, elle se trouve inscrite dans la légende sportive nationale, à l'instar du titre de champion du monde de football en 1998. Six mois après sa victoire, il est d'ailleurs élu par L'Équipe « champion des champions 1983 ». La prégnance de ce triomphe est telle que la mémoire collective en garde un souvenir vivace et que les médias ne se lassent pas, à chaque édition de Roland-Garros, de le commémorer. Depuis, aucun autre tennisman français n'a égalé sa performance. La victoire de la Franco-Américaine Mary Pierce en 2000 n'a pas laissé la même empreinte dans la 
mémoire nationale.Mais, en définitive, au-delà du champ sportif, sa portée est sans doute moindre que ce que certains augures prophétisaient en juin 1983. Bien avant le succès des footballeurs tricolores en 1998 et la mise en orbite du mythe de la France Black-Blanc-Beur, les médias croient voir dans son triomphe un "moment antiraciste ${ }^{64}$ ». L'at-il été ? Du point de vue médiatique, s'il a été, ce fut sur le mode du rêve, du fantasme, et il aura été aussi éphémère que fragile. La croyance dans les vertus pacificatrices et cathartiques du sport ne résiste pas à la vigueur des courants plus profonds qui ébranlent la société française en 1983. Ce n'est que plus tard que Noah affirme son charisme avec plus de force et de portée. Entre 2005 et 2007, il devient la personnalité préférée des Français (baromètre du Journal du dimanche). Depuis la fin de sa carrière sportive, il apparaît plus engagé contre les discriminations sociales (avec son association Fête le Mur) et on peut noter une stabilisation positive d'une africanité qu'il a lui-même mise en scène en tant que chanteur. Ainsi, en 1991, capitaine de l'équipe de France triomphante lors de la finale de la Coupe Davis, alors qu'il vient de mettre fin à près de soixante ans sans victoire dans cette compétition, il entraîne ses joueurs derrière lui dans un tour d'honneur, dansant sur son premier disque, Saga Africa. En entonnant ce grand succès populaire sur le court du Palais des sports de Lyon, à l'unisson avec le public, il réactive l'émotion collective de 1983 sur le mode de la double appartenance. Un symbole et, d'une certaine façon, l'annonce du mouvement plus tardif d'une France noire décomplexée ${ }^{65}$. Par la suite, les références médiatiques à la couleur de peau de Yannick Noah ne cessent de refluer. Dans le champ politique, on n'estime plus nécessaire de rappeler son métissage. Le 3 décembre 1996, alors qu'il vient de remporter une seconde Coupe Davis avec ses joueurs, Jacques Chirac a ce mot: "Yannick Noah, d'une certaine façon, a incarné la France, il était la France dans cette épreuve. »Si l'on questionne le " figement représentationnel ${ }^{66}$ » de la victoire de 1983, elle ne se rapporte pas à un moment antiraciste mais à une fulgurante décharge d'émotion et de gloire nationale. Ce qui amène, en dernier ressort, à revenir sur la question d'un héroïsme qui tend, sur le temps moyen, à estomper les couleurs pour mieux compenser le manque de modèle, d'émotions positives partagées ${ }^{67}$ dans une France hantée par l'idée de son déclin ${ }^{68}$. 